\title{
Philosophiques
}

\section{Kurt Lenk, Günter Meuter, Henrique Ricardo Otten, Les maîtres à penser de la Nouvelle Droite, traduit de l'allemand par Cécile Rol, Montréal, Liber, 2014}

\section{Christian Nadeau}

Volume 42, numéro 1, printemps 2015

URI : https://id.erudit.org/iderudit/1032228ar

DOI : https://doi.org/10.7202/1032228ar

Aller au sommaire du numéro

Éditeur(s)

Société de philosophie du Québec

ISSN

0316-2923 (imprimé)

1492-1391 (numérique)

Découvrir la revue

Citer ce compte rendu

Nadeau, C. (2015). Compte rendu de [Kurt Lenk, Günter Meuter, Henrique Ricardo Otten, Les maitres à penser de la Nouvelle Droite, traduit de l'allemand par Cécile Rol, Montréal, Liber, 2014]. Philosophiques, 42(1), 212-216.

https://doi.org/10.7202/1032228ar

Ce document est protégé par la loi sur le droit d'auteur. L'utilisation des services d'Érudit (y compris la reproduction) est assujettie à sa politique d'utilisation que vous pouvez consulter en ligne.

https://apropos.erudit.org/fr/usagers/politique-dutilisation/ 
Gibert. En fait, en évitant de trop se compromettre sur ces questions épineuses, ce dernier parvient à produire un portrait de l'imagination en morale qui saura rallier les éthiciens de diverses chapelles en leur donnant accès, dans un style clair, simple et agréable, à une documentation, des distinctions et des arguments qu'ils pourront adapter à leurs propres besoins.

BENOIT DUBREUIL

Kurt Lenk, Günter Meuter, Henrique Ricardo Otten, Les maîtres à penser de la Nouvelle Droite, traduit de l'allemand par Cécile Rol, Montréal, Liber, 2014.

On peut se réjouir de voir paraître cette traduction d'un ouvrage proposant une véritable synthèse de la pensée conservatrice et ce qu'on peut nommer la Nouvelle Droite. Il est permis de se demander ce qui a poussé un éditeur à traduire en français un livre paru en allemand en I997. La réponse se trouve pour partie dans l'absence d'étude sérieuse et systématique, en français, des origines de la pensée conservatrice. Chez les historiens, il s'agit moins de montrer la mécanique conceptuelle des idées que la propagation de celles-ci par différents mouvements sociaux. Pour ces théoriciens du politique, il s'agit de montrer la genèse intellectuelle d'une droite qui n'a que très peu à voir avec le libertarisme et qui au contraire attribue à celui-ci un rôle clef dans la promotion des valeurs traditionnelles ou imaginées comme telles.

Le but des auteurs ici, même s'ils en parlent en termes d'idéologie, n'est pas de proposer une description sociologique ni même historique de la Nouvelle Droite. Leur travail se présente plutôt sous la forme de courtes monographies où est analysée la contribution d'un auteur à ce qui est jugé au final comme un mouvement intellectuel. Certes, les auteurs se limitent à la première moitié du $\mathrm{Xx}^{\mathrm{e}}$ siècle. Il n'en demeure pas moins qu'ils ont à cœur de montrer la systématicité et la cohérence de thèses qui émergèrent en France notamment avec l'œuvre d'un Georges Sorel, pour ensuite se ramifier dans la pensée d'auteurs tels que Ernst Jünger ou de philosophes comme Martin Heidegger.

Les maîtres à penser de la Nouvelle Droite n'est pas, à proprement parler, un ouvrage de philosophie. Il est toutefois indispensable à celles et ceux qui, parmi les philosophes, cherchent à comprendre une pensée qui, tout en s'affichant résolument hostile au libertarisme et au capitalisme, s'oppose également au socialisme et à la justice sociale, et à toute forme de compromis obtenu par la médiation des institutions démocratiques. Les institutions de l'État jouent un rôle central, mais à la condition qu'elles représentent une autorité supérieure aux individus. La Nouvelle Droite prétend en fait offrir au peuple la véritable voie, où celui-ci verrait niée l'universalité des droits au profit de l'identité des nations. Les objectifs d'un tel programme réclament un abandon total des individus et de leur société à 
une collectivité politique homogène, qui fait bloc, et qui donc se doit de refuser le pluralisme et le débat démocratique. La haine du capitalisme est associée à celle à l'égard de l'individualisme bourgeois pour culminer dans une critique fondamentale de la "décadence» des sociétés modernes. La Nouvelle Droite représente bien le discours conservateur tel qu'on peut l'entendre encore aujourd'hui, moins chez les politiciens que chez un certain nombre d'intellectuels et de philosophes, surtout en Europe.

Le premier chapitre expose les thèses fondatrices de la Nouvelle Droite, que les auteurs retrouvent dans la pensée de Georges Sorel, dont l'héritage intellectuel est pour le moins controversé. Dans ses œuvres, Sorel oppose la simplicité directe du monde paysan au discours technique et arrogant des défenseurs de la modernité. L'idée de décadence morale apparaît dès lors qu'une séparation s'opère entre le monde de la nature et celui de la culture, par exemple lorsque le contrat social l'emporte sur la communauté familiale comme socle de la société. Chez Sorel, le contenu propre au discours d'une communauté politique compte moins que la force dont elle dispose pour se faire entendre, et la force ici se vérifie dans l'enracinement des pratiques sociales. L'ensemble des notions morales sont dès lors vues comme de simples constructions sociales qui ne correspondent en rien aux mœurs, à la culture et à l'identité des peuples. Lorsqu'un auteur comme Sorel se penche sur la corruption, ce n'est pas la stabilité des institutions qui l'intéresse, mais l'unité de la communauté politique vers une seule et même visée. La corruption est alors signe de décadence: la vertu du collectif s'épuise pour laisser place aux intérêts particuliers. Sorel dénoncera donc la démocratie en ellemême, et non ses dérives ou ses déboires: la démocratie en elle-même, par son égalitarisme, ne peut échapper de manière structurelle à la duplicité et au mensonge électoraliste. Associé au socialisme, Sorel se révèle aux yeux de Lenk, Meuter et Ricardo Otten, un apologiste de la lutte des classes où celleci représente un état de guerre. Sorel introduit alors deux éléments qui seront constitutifs du discours de la Nouvelle Droite: le mythe social et la violence qui l'accompagne. Le mythe est seul capable de mobilisation sociale, et la violence est son expression paradigmatique, à l'encontre de la raison qui par définition demande le dialogue et donc la pacification des rapports sociaux.

Le deuxième chapitre analyse la pensée d'Oswald Spengler, l'auteur du fameux livre Le déclin de l'Occident, qui encore aujourd'hui tient lieu de référence à de nombreux auteurs. L'idée de «déclin » ici se voit associée à la montée du libéralisme et de la démocratie. Nulle nostalgie pour un monde perdu chez Spengler. Il s'agit d'abord et avant tout d'un constat: le monde ancien devait périr par la technique, il le méritait pour ainsi dire. Le propos normatif de Spengler est ailleurs: si ce monde devait mourir, c'est qu'il faut le remplacer par un autre. À l'encontre de ce que l'on retrouvera chez Heidegger, la technique, le monde des machines propre à la société industrielle $\mathrm{du} \mathrm{XX}^{\mathrm{e}}$ siècle représente une force de transformation radicale du monde. Au delà des idéologies, Spengler en appelle à un socialisme dénué de tout projet 
politique, qui serait d'abord et avant tout un état moral, un éthos propre à une communauté donnée.

Le troisième chapitre porte sur l'une des figures les moins connues de la Nouvelle Droite par le public francophone, Hans Freyer. Tout comme Spengler et Sorel, Freyer refuse les médiations de la démocratie et le pluralisme libéral pour lui préférer la domination du peuple, vu comme sujet politique dont la cohésion dépend d'une "croyance» commune comme ciment des relations sociales. Au delà de la puissance d'une unité, le peuple doit exercer, dit Freyer, une véritable domination, sans quoi il ne peut être libre.

Le quatrième chapitre montre la contribution essentielle de l'auteur certainement le plus connu, le théoricien du droit Carl Schmitt (pour le public francophone, de très nombreux livres de Schmitt sont maintenant disponibles en français). La bête noire de Schmitt fut certainement le libéralisme, qui inverse le rapport axiologique entre l'individu et l'État et fait de celui-ci le protecteur de celui-là. Pour Schmitt, l'État est au contraire seul à même d'élever les individus à «la condition d'êtres moraux» (p. 98). L'État occupe la plus haute position hiérarchique, car il agit comme un aimant qui guide les aspirations de tout membre de la communauté politique. L'État est pour Schmitt «seul sujet de droit» (ibid.). Dès lors, si la démocratie peut avoir un sens, c'est uniquement en ce qu'elle représente une identité parfaite entre gouvernants et gouvernés. C'est la raison pour laquelle d'ailleurs bon nombre d'auteurs de gauche, à commencer par le philosophe Ernesto Laclau, prétendent aujourd'hui s'inspirer de Schmitt. Or, chez ce dernier, l'identification des gouvernés aux gouvernants n'implique en rien un accroissement des pouvoirs des membres de la communauté politique. Dès lors, la seule véritable option possible pour le peuple est l'acclamation des décisions prises en leur nom. Le principal rôle des institutions est justement de décider — d'où l'importance chez Schmitt du paradigme de l'état d'exception -, en particulier lorsqu'il faut défendre l'unité politique de l'État par l'affirmation de sa puissance.

Le chapitre V est consacré à l'œuvre de Martin Heidegger. Il faut rappeler que Les maîtres à penser de la Nouvelle Droite a été écrit avant la publication des fameux "Cahiers noirs" où l'antisémitisme de Heidegger apparaît de manière flagrante et s'inscrit au cœur de sa démarche philosophique. C'est peut-être chez Heidegger que l'on comprend le mieux la critique radicale d'une participation à la vie politique démocratique, celle-ci étant le lieu d'une perte de sens, d'une dispersion qui éloigne les individus de leur authenticité. Inversement, l'individu s'accomplit dans la nation ou, pour reprendre les termes de Heidegger lui-même, «l'aventure de la communauté du peuple» (p. I32). Si beaucoup de choses ont été écrites sur le rapport de Heidegger au nazisme, ce chapitre offre des perspectives nouvelles en ce qu'il montre la contribution spécifique de l'auteur d'Être et temps à la formation du paradigme conservateur à son époque ainsi que ses influences. 
Le sixième et dernier chapitre présente l'œuvre de l'écrivain Ernst Jünger comme un aboutissement logique de la Nouvelle Droite, et plus particulièrement la fascination de la violence. Là encore, l'unité de la communauté politique se manifeste par sa capacité d'action, celle-ci ne pouvant se trouver entravée par des états d'âme ou par des disputes politiques. Ce qui, selon Jünger, distingue la Révolution russe d'octobre de la révolution allemande qui a conduit à la création de la République de Weimar est que la première a affirmé son existence et sa volonté de puissance par la violence. On a souvent identifié la formation intellectuelle du nazisme en Allemagne au mouvement romantique. Or, ni Schmitt ni Spengler ni Jünger ne se réclament du romantisme: au contraire, pour ces conservateurs, qui en ce sens se démarquent de nombreux protagonistes intellectuels de l'autoritarisme politique, le romantisme est trop marqué par une négation des choses dont il peine à sortir. La négation du monde est la condition de sa transformation, pense Jünger, d'où le recours encore une fois à une figure mythique, celle du «travailleur» qui détruit l'ordre ancien pour en créer un nouveau. Mais si tel est le cas, peuton réellement parler de conservatisme? Oui, si la destruction de l'ordre ancien correspond en réalité à celle de la modernité, laquelle serait un obstacle à une quête d'authenticité puisqu'elle entrave l'existence de médiations, celles de la politique, de la société, des destins individuels. Si, vers la fin de sa vie, Jünger se fait l'apologue de l'individu, c'est que pour lui, au final, masse et individualité sont les deux faces d'une même réalité (p. I 58 ) : en se dépossédant de ce qui l'encombre, l'individu fait le sacrifice de son existence pour la seule chose qui soit à même de l'élever, une communauté qu'il n'ose plus qualifier de nation.

L'analyse philosophique ou théorique est privilégiée dans ce livre au détriment des considérations contextuelles. Le lecteur apprendra peu de choses sur - pour ne donner qu'un exemple — le rôle de Carl Schmitt à la fin de la République de Weimar et dans la création de l'ordre nouveau nazi. On peut comprendre un tel pari méthodologique de la part de Lenk, Meuter et Otten s'ils ont voulu empêcher toute réduction des idées aux simples actes de Freyer ou encore de Spengler. Mais un vrai travail d'histoire des idées nécessitait d'accorder une place plus importante au contexte de réception de leurs œuvres. D'un autre côté, le livre n'offre pas non plus d'analyse systématique de la Nouvelle Droite, au point qu'il est parfois difficile d'y voir un mouvement intellectuel. Les liens entre les auteurs sont plus proches de l' " air de famille ", même pour certaines idées, les filiations et les dettes respectives sont faciles à identifier. Il est dommage que Lenk, Meuter et Otten n'aient pas ajouté un chapitre de conclusion permettant une meilleure vue d'ensemble.

Les maîtres à penser de la Nouvelle Droite n'est pas un livre facile d'accès pour au moins deux raisons. En premier lieu, le livre propose un survol très rapide des auteurs étudiés, mais au prix d'un texte extrêmement dense. En second lieu, malgré un pari pédagogique évident, Lenk, Meuter et Otten ont tendance à remplacer l'analyse textuelle par des formules lapidaires qui 
ne sont pas toujours très claires, formules parfois même un peu sur le modèle des auteurs qu'ils critiquent. Cela étant dit, il s'agit d'un livre important, et toute analyse philosophique sérieuse des critiques du libéralisme et du discours théorique du conservatisme devra tenir compte de cette contribution très utile.

CHRISTIAN NADEAU

Université de Montréal

Jacqueline Mowbray. Linguistic Justice: International Law and Language

Policy, Oxford University Press, Oxford, 2012, 227 p.

Philippe Van Parijs. Linguistic Justice for Europe and for the World, Oxford

University Press, Oxford, 2011, 299 p.

\section{La justice linguistique}

Le débat portant sur la justice linguistique est une extension récente de la recherche portant sur l'application des théories de la justice dans les contextes de diversité culturelle. Si quelques propositions normatives dans le domaine des politiques linguistiques ont été mises de l'avant dans les années I990, il nous aura fallu attendre vingt ans avant que soient offertes des tentatives de formulation de théories normatives générales visant à identifier les principes de justice qui devraient orienter les politiques linguistiques et organiser les institutions chargées de gérer la diversité linguistique, ou ayant un effet sur cette diversité.

Je propose ici la recension de deux ouvrages importants publiés à quelques mois d'intervalle. Le premier à avoir été publié est l'œuvre du philosophe politique Philippe Van Parijs, Linguistic Justice for Europe and for the World, dans lequel il reprend les arguments articulés et développés dans plusieurs articles. Il y propose une version complète et cohérente de ses arguments et ajoute un élément jusque là largement ignoré par lui: la reconnaissance de l'aspect identitaire associé aux langages. Le second ouvrage est celui de la juriste Jacqueline Mowbray, intitulé Linguistic Justice: International Law and Language Policy. Dans cet ouvrage, l'auteure propose une évaluation critique du droit international en matière linguistique. Elle y évalue autant les institutions chargées explicitement de protéger ou de promouvoir la diversité linguistique que les autres outils juridiques ayant un effet sur la diversité linguistique.

Les deux auteurs abordent la question de la justice linguistique à partir d'angles distincts mais complémentaires. Mowbray aborde la question de la justice linguistique à partir d'une analyse des relations de pouvoir, des structures sociales désavantageant systématiquement les locuteurs minoritaires. Ces désavantages font que les locuteurs minoritaires souffrent d'un déficit de 